\title{
Bacillus Pumilus Septicemia: A Neonatal Case
}

\author{
F. Bennaoui ${ }^{1,2}$, N.EL Idrissi Slitine ${ }^{1,2}$, Kouta Rabii ${ }^{1,2}$, N. Soraa ${ }^{3}$ FMR. Maoulainine ${ }^{1,2}$ \\ ${ }^{1}$ Neonatal Intensive Care Unit, Mohammed VI University Hospital Center, Marrakech, Morocco \\ ${ }^{2}$ Research laboratory: Heath, children and development, Faculty of Medicine and Pharmacy of \\ Marrakech, Cadi Ayyad University Marrakech Morocco \\ ${ }^{3}$ Department of Microbiology, Mohammed VI University Hospital Center, Marrakech, Morocco \\ *Corresponding Author: F. Bennaoui, Neonatal Intensive Care Unit, Mohammed VI University \\ Hospital Center, Marrakech, Morocco.
}

\begin{abstract}
Bacillus Pumilus (B. Pumilus) is an environmental contaminant rarely associated with clinical infections. Here, we report a case of severe septicemia caused by B. pumilus identified in a term newborn; a newborn male without any factor predisposing to infection; he is hospitalized for jaundice complicated by nosocomial infection. In this case, the microorganism was detected from repeated blood cultures. The newborn was treated with imipenem, amikacin and vancomycin with a fatal outcome. This report reveals the potential role of B. pumilus as a pathogen in the bloodstream during infancy.
\end{abstract}

Keywords: Baciluspumilus, septicemia, blood culture

\section{INTRODUCTION}

Bacillus pumilus is a Gram-positive, aerobic, spore-forming microrganism usually found in the soil as a commensal and more commonly isolated in cultures as contaminant. The clinical significance of the isolation of these microorganisms which produce a careful assessment, the initial report of a blood culture growing a species of Bacillus, often creates a therapeutic dilemma. Other than Bacillus anthracis, species of the genus Bacillus are rarely associated with infection, despite their widespread distribution in the environment, and are more frequently isolated as contaminants of culture[1]. Bacteremia caused by Bacillus species is mainly attributed to Bacillus cereus and has been reported mainly in immunocompromised patients with hematologic malignancies [2] [3][4].

Here, we report a rare case in the literature of severe sepsis caused by Bacillus pumilus in a newborn no having a predisposing factors.

\section{Case Report}

Anewborn male, born from a twin pregnancy, presenting a harmonious intrauterine growth retardation with a weight of $1300 \mathrm{~g}$. His mother had 21 year old, primiparous without any chronique disease or infectionat term (gestation of 39 weeks).
On the 9th postnatal day, the newborn presented with neonatal jaundice, associated with hypertonic seizures without other clinical signs of infection. The initial biological assessment objectified leukopenia at $3540 / \mu 1$, thrombo cytopenia at $123,000 / \mu 1$ with a CRP at 2.92 $\mathrm{mg} / \mathrm{l}$ and a negative respiratory PCR. However, the infant complicated by septicemia 2 days after admission and presented with respiratory distress for which he had to be intubated ventilated and sedated.

Laboratory analysis showed a white blood cell count of 23,600 / mm3 and a high level of Creactive protein $(110 \mathrm{mg} / \mathrm{l})$. The cultures of urine and cerebrospinal fluid (CSF) were sterile. Blood culture has shown growth of Grampositive rods in chains. Subsequently, the microorganism was identified as Bacillus Pumilus.

The newborn was treated by Imipenem, Amikacin and Vancomycin.The evolution was marked by worsening respiratory distress and the occurrence of the death of the newborn after 10 days.

\section{Discussion}

Bacillus species, apart from B. anthracis and B. cereus, havelittle or no pathogenic potential and are rarely associated with clinical infections (Drobniewski, 1993)[4]. A computerized search in the National Library of Medicine database provided a total of 18 cases of clinically 
significant infections caused by B. pumilus. These infections fall into three broad groups: (i) 10 cases of bloodstream infections (nine cases in adults and one case in an 8-year-old child) $[2][5][3][6][7][8][9]$; (ii) three cases of cutaneous infections [10]; and (iii) five cases of food poisoning, characterized by toxin-induced emetic and diarrheagenic syndromes [11].

The present study describes, one of the rarest cases of septicemia caused by B. pumilus in neonates. Documented cases of clinically significant bloodstream infections in neonates or older infants due to non-anthracis Bacillus species are very limited and include only cases caused by B. cereus [2][12]. Maria Kimoul and al. report two cases of severe sepsis caused by Bacillus pumilus in neonatal infants successfully treated with vancomycin[13].This is despite the fact that the neonatal population is particularly susceptible to disseminated disease caused by environmental organisms due to molecular, cellular and functional deficiency of both cellular and humoral immunity. The low number of cases could be partially attributed to the fact that clinical laboratories may not attempt to identify Bacillus organisms at the species level, arbitrarily designating them as contaminants, without adequate consultation with clinicians. Previous studies have shown that Bacillus species should be recognized as true pathogens, especially in neonates and other immuno suppressed hosts and when isolated from blood cultures collected at the same time or from at least two samples collected at different time [5]. In our case, B. pumilus was identified in a blood culture, which in combination with an obvious clinical picture, meets the appropriate criteria to distinguish a real infection of the blood from a simple stain in the sample.

Risk factors for B. pumilus bacteremia in adults have included use of a central venous catheter [8] cancer [3][9], particularly hematological malignancies [5][7], and spinal anesthesia [2]. The predisposing factors identified in our newborn are: mechanical ventilation, umbilical venous catheterization and the use of long-term intravascular catheters.

B. pumilus isolated from our new patient was susceptible to penicillin, vancomycin and the vast majority of other antibiotics tested.Both neonates promptly responded to vancomycin treatment, which is considered the drug of choice for Bacillus infections.
In conclusion, our study highlights the fact that B. pumilus is a potential bloodstream pathogen in neonates, and should be recognized as such, especially if this micro-organism is isolated from subsequent blood cultures.

\section{REFERENCES}

[1] Logan, N. A., Popovic, T. \& Hoffmaster, A. (2009). Bacillus and other aerobic endosporeforming bacteria. In Manual of Clinical Microbiology, 9th edn, pp. 455-473. Edited by P. R. Murray, E. J. Baron, J. H. Jorgensen, M. L. Landry \& M. A. P faller. Washington, DC: American Society for Microbiology.

[2] Farrar, W. E., Jr (1963). Serious infections due to "non-pathogenic" organisms of the genus Bacillus. Review of their status as pathogens. Am J Med 34, 134-141.

[3] Banerjee, C., Bustamante, C. I., Wharton, R., Talley, E. \& Wade, J. C. (1988). Bacillus infections in patients with cancer. Arch Intern Med 148, 1769-1774.

[4] Drobniewski, F. A. (1993). Bacillus cereus and related species. Clin Microbiol Rev 6, 324-338.

[5] Cotton, D. J., Gill, V. J., Marshall, D. J., Gress, J., Thaler, M. \& Pizzo, P. A. (1987). Clinical features and therapeutic interventions in 17 cases of Bacillus bacteremia in an immunosuppressed patient population. J ClinMicrobiol 25, 672-674.

[6] Galanos, J., Perera, S., Smith, H., O’Neal, D., Sheorey, H. \& Waters, M. J. (2003). Bacteremia due to three Bacillus species in a case of Munchausen's syndrome. J ClinMicrobiol 41, 2247-2248.

[7] Ozkocaman, V., Ozcelik, T., Ali, R., Ozkalemkas, F., Ozkan, A., Ozakin, C., Akalin, H., Ursavas, A., Coskun, F. \& other authors (2006). Bacillus spp. among hospitalized patients with haematological malignancies: clinical features, epidemics and outcomes. J Hosp Infect 64, 169-176.

[8] Bentur, H. N., Dalzell, A. M. \& Riordan, F. A. I. (2007). Central venous catheter infection with Bacillus pumilus in an immunocompetent child: a case report. Ann Clin Microbiol Antimicrob 6, 12.

[9] Farhat, H., Chachaty, E., Antoun, S., Nitenberg, G. \& Zahar, J.-R. (2008). [Two cases of Bacillus infection and immunodepression]. Med Mal Infect 38, 612-614 (in French).

[10] Tena, D., Martinez-Torres, J. A., Perez-Pomata, M. T., Sa'ez-Nieto, J. A., Rubio, V. \& Bisquert, J. (2007). Cutaneous infection due to Bacillus pumilus: report of 3 cases. Clin Infect Dis 44, e40-e42.

[11] From, C., Hormazabal, V. \& Granum, P. E. (2007). Food poisoning associated with 
pumilacidin-producing Bacillus pumilus in rice. Int J Food Microbiol 115, 319-324.

[12] Adler, A., Gottesman, G., Dolfin, T., Arnon, S., Regev, R., Bauer, S. \& Litmanovitz, I. (2005). Bacillus species sepsis in the neonatal intensive care unit. J Infect 51, 390-395.
[13] Maria K., Georgia V., Magdalini P., Vasiliki K., Dimitra P., Antonios G., Alexander W. F.and Athanassios T. Two cases of severe sepsis caused by Bacilluspumilus in neonatal infants Journal of Medical Microbiology (2012), 61, 596-599.

Citation: F. Bennaoui et al, “Bacillus Pumilus Septicemia: A Neonatal Case”, International Journal of Research Studies in Medical and Health Sciences. 2021; 6(3): 18-20. DOI: https://doi.org/10.22259/ijrsmhs. 0603004

Copyright: (C) 2021 F. Bennaoui et al, This is an open-access article distributed under the terms of the Creative Commons Attribution License, which permits unrestricted use, distribution, and reproduction in any medium, provided the original author and source are credited. 